TRANSACTIONS OF THE

AMERICAN MATHEMATICAL SOCIETY

Volume 354, Number 10, Pages 3893-3906

S 0002-9947(02)03072-6

Article electronically published on June 5, 2002

\title{
UNIVERSAL DEFORMATION RINGS AND KLEIN FOUR DEFECT GROUPS
}

\author{
FRAUKE M. BLEHER
}

\begin{abstract}
In this paper, the universal deformation rings of certain modular representations of a finite group are determined. The representations under consideration are those which are associated to blocks with Klein four defect groups and whose stable endomorphisms are given by scalars. It turns out that these universal deformation rings are always subquotient rings of the group ring of a Klein four group over the ring of Witt vectors.
\end{abstract}

\section{INTRODUCTION}

Suppose $k$ is a field of positive characteristic $p, W=W(k)$ is the ring of infinite Witt vectors over $k$, and $G$ is a profinite group. In [16], 17], Mazur developed a deformation theory of finite-dimensional representations $V$ of $G$ in case $k$ is finite, using results of Schlessinger [21. He proved that if $G$ satisfies a certain finiteness condition, then $V$ has a universal deformation $\operatorname{ring} R(G, V)$ in case $V$ is absolutely irreducible. In 12, de Smit and Lenstra used an argument of Faltings to prove for arbitrary profinite groups $G$ that a finite-dimensional representation $V$ of $G$ over $k$ has a universal deformation $\operatorname{ring} R(G, V)$ in case the endomorphism ring of $V$ is just $k$. The $\operatorname{ring} R(G, V)$ is defined as follows. Suppose $\mathcal{C}$ is the category of all topological local commutative $W$-algebras $R$ with residue field $k$ which are the projective limits of their discrete Artinian quotients. Then a lift of $V$ over an object $R$ in $\mathcal{C}$ is an $R G$-module $M$ which is free over $R$ so that $k \otimes_{R} M \cong V$ as $k G$-modules. An isomorphism class of lifts of $V$ over $R$ is called a deformation of $V$ over $R$. The deformation functor $\mathcal{F}_{V}: \mathcal{C} \rightarrow$ Sets sends an object $R$ in $\mathcal{C}$ to the set of all deformations of $V$ over $R$. Then $V$ has a universal deformation ring $R(G, V)$ in $\mathcal{C}$ if the functor $\mathcal{F}_{V}$ is naturally isomorphic to $\operatorname{Hom}_{\mathcal{C}}(R(G, V)$, - ); in other words, if $R(G, V)$ represents $\mathcal{F}_{V}$. For more information on deformations and deformation rings, see [12] and [16]. In number theory, deformation rings are at the center of work by many authors concerning Galois representations, modular forms, elliptic curves and diophantine geometry (see, for example, [25], [23, [6], [9], and their references).

Suppose now that $k$ is algebraically closed. To answer questions about the ring structure of universal deformation rings, it is natural to start with the case of a finite group $G$. In $[3$, the universal deformation ring of a representation $V$ of a finite group $G$ over $k$ has been determined explicitly in case the stable endomorphism ring

Received by the editors October 23, 2001 and, in revised form, April 2, 2002.

2000 Mathematics Subject Classification. Primary 20C05; Secondary 16G10.

Key words and phrases. Universal deformation rings, Klein four groups, tame blocks.

The author was supported in part by NSA Young Investigator Grant MDA904-01-1-0050. 
of $V$ is $k$ and the unique non-projective indecomposable summand of $V$ belongs to a block of $k G$ with cyclic defect groups. The obtained isomorphism types of $R(G, V)$ in this case lead to the following general question.

Question 1.1. Suppose $G$ is a finite group and $V$ is a finitely generated $k G$-module with stable endomorphism ring $k$. Is $R(G, V)$ a subquotient ring of the group ring $W D$ over $W$ of a defect group $D$ of the block $B$ of $k G$ associated to $V$ ?

In [3], it follows that this question has a positive answer in case $D$ is cyclic. In this paper we prove:

Theorem 1.2. Question 1.1 has a positive answer in case $D$ is a Klein four group.

This case provides a more stringent test of Question 1.1 than the cyclic block case in [3], since the representation theory of a block $B$ with Klein four defect group $D$ is considerably more difficult. For example, there are infinitely many non-isomorphic indecomposable $B$-modules, some of which are parametrized by one-parameter families, whereas a cyclic block has only finitely many indecomposable modules up to isomorphism. It is also more difficult to determine all the indecomposable $B$-modules which have stable endomorphism ring $k$; this was relatively easy to determine for cyclic blocks. We prove Theorem 1.2 by determining explicitly all the universal deformation rings.

Theorem 1.2 supports the idea that Question 1.1 is the correct question to consider concerning the dependence of the universal deformation rings $R(G, V)$ on the local structure of $G$. In case $D$ is non-abelian, it is natural to ask if Question 1.1 can be refined, since $W D$ is no longer a commutative ring. One possibility would be to replace $D$ by its maximal abelian quotient. It turns out that this is not possible in general. A counterexample is given when $k$ has characteristic $2, G$ is the symmetric group $S_{4}$, and $V$ is an irreducible 2-dimensional representation of $G$ over $k$ (see Proposition 4.2 and Remark 4.4). Note that in this case the defect group $D$ is a dihedral group of order 8 .

To analyze Question 1.1 for more complicated defect groups, a case-by-case study as done in this paper is inefficient and often not possible. In case $D$ is abelian, there are strong conjectures posed by Broué and others (see, e.g., [7, [19]) which would establish derived equivalences between blocks of $k G$ with defect group $D$ and associated blocks of $k N_{G}(D)$. Such equivalences should be useful in studying Question 1.1 when $D$ is abelian. Issues of this kind arise in Remark 3.7 of this paper. In particular, Rickard has shown in [18 that there is a splendid derived equivalence between the principal blocks of the alternating groups $A_{4}$ and $A_{5}$ when $p=2$. In this paper, we determine the universal deformation rings of all $V$ belonging to these blocks over $k$ and having stable endomorphism ring equal to $k$. It is a natural problem now to find whether these calculations are consistent with universal deformation rings being preserved by (splendid) derived equivalences. As a first step to study this problem, Mazur's deformation theory has been extended in [5] to objects in derived categories.

The sections of this article are as follows. In Section 2, we recall some basic definitions and results. In particular, Proposition 2.5 shows that Morita equivalences between blocks of group rings over $W$ preserve universal deformation rings. In Section 3, we prove Theorem[1.2 using a case-by-case analysis. In Section 4, we determine the universal deformation rings of the irreducible representations of $k S_{4}$ in case the characteristic of $k$ is 2 . 
I would like to thank the referee for his or her comments that helped improve the final version of this paper.

Throughout the paper, $k$ is an algebraically closed field of characteristic $p>0$ and $G$ is a finite group.

\section{Preliminaries}

In this section, we recall a few basic definitions and results and prove that Morita equivalences between blocks of group rings over $W$ preserve universal deformation rings. As in Section 1 , let $\mathcal{C}$ be the category of all topological local commutative $W$-algebras $R$ such that $R / \max (R)=k$ and $R$ is the projective limit of its discrete Artinian quotients. The morphisms in $\mathcal{C}$ are continuous $W$-algebra homomorphisms. Suppose $G$ is a finite group and $V$ is a finitely generated $k G$-module. Recall from Section 1 that a lift of $V$ over an object $R$ in $\mathcal{C}$ is an $R G$-module $M$ which is free over $R$ so that $k \otimes_{R} M$ is isomorphic to $V$ as a $k G$-module. The isomorphism class [ $M]$ of $M$ as an $R G$-module is called a deformation of $V$ over $R$, and the set of such deformations is denoted by $\operatorname{Def}_{G}(V, R)$. The deformation functor $\mathcal{F}_{V}: \mathcal{C} \rightarrow$ Sets sends an object $R$ in $\mathcal{C}$ to $\operatorname{Def}_{G}(V, R)$ and a morphism $f: R \rightarrow R^{\prime}$ in $\mathcal{C}$ to the map $\operatorname{Def}_{G}(V, R) \rightarrow \operatorname{Def}_{G}\left(V, R^{\prime}\right)$ defined by $[M] \mapsto\left[R^{\prime} \otimes_{R, f} M\right]$. In case there exists an object $R(G, V)$ in $\mathcal{C}$ and a lift $U(G, V)$ of $V$ over $R(G, V)$ so that for each $R$ in $\mathcal{C}$ and for each lift $M$ of $V$ over $R$ there is a unique morphism $\alpha: R(G, V) \rightarrow R$ in $\mathcal{C}$ with $[M]=\left[R \otimes_{R(G, V), \alpha} U(G, V)\right]$, then $R(G, V)$ is called the universal deformation ring of $V$ and $[U(G, V)]$ is called the universal deformation of $V$. In other words, $R(G, V)$ represents the functor $\mathcal{F}_{V}$ in the sense that $\mathcal{F}_{V}$ is naturally isomorphic to $\operatorname{Hom}_{\mathcal{C}}(R(G, V),-)$. By a result of Faltings (see 12, Prop. 7.1]), $V$ has a universal deformation ring $R(G, V)$ in case $\operatorname{End}_{k G}(V)=k$.

For the convenience of the reader, we now recall some results from [3]. Suppose $\Omega$ denotes the Heller operator for $k G$ (see, for example, [1 §20]). Define $d^{j}(V)=\operatorname{dim}_{k} \hat{H}^{j}\left(G, \operatorname{Hom}_{k}(V, V)\right)$ for $j \in \mathbb{Z}$, where $\hat{H}^{j}$ denotes the $j$-th Tate cohomology group. In particular, $\hat{H}^{0}\left(G, \operatorname{Hom}_{k}(V, V)\right)=\underline{\operatorname{End}}_{k G}(V)$, and for $j>0$, $\hat{H}^{j}\left(G, \operatorname{Hom}_{k}(V, V)\right)=\operatorname{Ext}_{k G}^{j}(V, V)$.

Proposition 2.1 ([3 Prop. 2.1]). Suppose $V$ is a finitely generated $k G$-module with $d^{0}(V) \leq 1$. Then $V$ has a Noetherian universal deformation ring $R(G, V)$.

Lemma 2.2 ([3, Cor. 2.5]). Let $V$ be a finitely generated $k G$-module.

(i) There is an isomorphism $\underline{\operatorname{End}}_{k G}(V) \cong \underline{\operatorname{End}}_{k G}(\Omega(V))$ of $k$-vector spaces. In particular, $d^{0}(V)=d^{0}(\Omega(V))$.

(ii) If $d^{0}(V) \leq 1$, then $R(G, V)$ and $R(G, \Omega(V))$ are isomorphic.

(iii) If $d^{0}(V)=0$, then $R(G, V)=W$.

Lemma 2.3 ([3, Cor. 2.8]). Suppose $V$ is a finitely generated $k G$-module with $d^{0}(V)=1$. Then there is a non-projective indecomposable $k G$-module $V_{0}$ such that $d^{0}\left(V_{0}\right)=1, V$ is isomorphic to $V_{0} \oplus P$ for some projective $k G$-module $P$ and $R(G, V) \cong R\left(G, V_{0}\right)$.

Remark 2.4. Suppose $V$ is a 1-dimensional representation of $G$ over $k$. According to Mazur [16], the universal deformation ring of $V$ is $R(G, V)=W G^{a b, p}$, where $G^{a b, p}$ is the maximal abelian $p$-quotient of $G$. Similarly to [1. pp. 67-68], it follows that the vertices of $V$ are Sylow $p$-subgroups of $G$. This implies that the defect groups of the block to which $V$ belongs are also Sylow $p$-subgroups of $G$. Hence Question 1.1 has a positive answer in case $V$ is 1-dimensional over $k$. 
We now show that universal deformation rings are preserved by Morita equivalences between blocks of group rings over $W$. Since every block of $k G$ can be lifted to a block of $W G$, every finitely generated indecomposable $k G$-module belongs to a unique block of $W G$.

Proposition 2.5. Let $V$ be a finitely generated indecomposable $k G$-module with $d^{0}(V)=1$ so that $V$ belongs to the block $B_{W}$ of $W G$. Suppose $B_{W}$ is Morita equivalent to a block $b_{W}$ of $W H$ for some finite group $H$, and $V^{\prime}$ is the $k H$-module corresponding to $V$ under this Morita equivalence. Then $R(G, V)$ and $R\left(H, V^{\prime}\right)$ are isomorphic.

Proof. Suppose the Morita equivalence is given as

$$
Q \otimes_{b_{W}}-: b_{W}-\bmod \rightarrow B_{W} \text {-mod }
$$

where $Q$ is a $B_{W}-b_{W}$-bimodule which is a finitely generated projective generator of $B_{W}$-mod, and $\operatorname{End}_{B_{W}}(Q) \cong b_{W}^{o p}$. Let $R \in \mathrm{Ob}(\mathcal{C})$, and let $B_{R}$ (respectively $b_{R}$ ) be the block of $R G$ (respectively of $R H$ ) corresponding to $B_{W}$ (respectively to $\left.b_{W}\right)$. Then $B_{R}=R \otimes_{W} B_{W}$ and $b_{R}=R \otimes_{W} b_{W}$. It follows that $R \otimes_{W} Q$ is a $B_{R}$ - $b_{R}$-bimodule which is a finitely generated projective generator of $B_{R}$-mod. We now show that $\operatorname{End}_{B_{R}}\left(R \otimes_{W} Q\right) \cong b_{R}^{o p}$ by showing that $\operatorname{End}_{R G}\left(R \otimes_{W} Q\right) \cong$ $R \otimes_{W} \operatorname{End}_{W G}(Q)$ as $R$-algebras. Since $W$ is a principal ideal domain, it can easily be seen that

$$
\operatorname{End}_{k G}\left(k \otimes_{W} Q\right) \cong k \otimes_{W} \operatorname{End}_{W G}(Q)
$$

as $k$-algebras. We define an $R$-algebra homomorphism

$$
\beta: R \otimes_{W} \operatorname{End}_{W G}(Q) \rightarrow \operatorname{End}_{R G}\left(R \otimes_{W} Q\right)
$$

by

$$
r \otimes f \mapsto \operatorname{mult}(r) \otimes f .
$$

Using (2.2), it follows that $\beta$ induces an isomorphism

$$
\operatorname{id} \otimes \beta: k \otimes_{R} R \otimes_{W} \operatorname{End}_{W G}(Q) \rightarrow k \otimes_{R} \operatorname{End}_{R G}\left(R \otimes_{W} Q\right) .
$$

Because $Q$ is a summand of a free $W G$-module of finite rank, $R \otimes_{W} Q$ is a summand of a free $R G$-module of finite rank. Since $G$ is finite and $W$ and $R$ are objects in $\mathcal{C}$, it follows that $\operatorname{End}_{W G}(Q)$ (respectively $\left.\operatorname{End}_{R G}\left(R \otimes_{W} Q\right)\right)$ is a finitely generated free $W$-module (respectively a finitely generated free $R$-module). Thus $\beta$ is an $R$-algebra isomorphism. Hence (2.1) induces a Morita equivalence

$$
\left(R \otimes_{W} Q\right) \otimes_{b_{R}}-: b_{R}-\bmod \rightarrow B_{R}-\bmod
$$

and $V \cong\left(R \otimes_{W} Q\right) \otimes_{b_{R}} V^{\prime}$. Suppose now that $M^{\prime}$ is a lift of $V^{\prime}$ over $R$. Then $M^{\prime}$ is a $b_{R}$-module. Since $M^{\prime}$ is finitely generated free over $R$ and $R \otimes_{W} Q$ is projective over $b_{R}$, it follows that $M=\left(R \otimes_{W} Q\right) \otimes_{b_{R}} M^{\prime}$ is finitely generated free over $R$. Moreover, $M \otimes_{R} k=\left(R \otimes_{W} Q\right) \otimes_{b_{R}}\left(M^{\prime} \otimes_{R} k\right) \cong\left(R \otimes_{W} Q\right) \otimes_{b_{R}} V^{\prime} \cong V$. Hence $M$ is a lift of $V$. We thus obtain a bijection between the deformations of $V^{\prime}$ over $R$ and the deformations of $V$ over $R$. This implies that the two deformation functors $\mathcal{F}_{V^{\prime}}$ and $\mathcal{F}_{V}$ are naturally isomorphic, which completes the proof of Proposition 2.5. 


\section{Blocks With KLEIN FOUR DEFECT GROUPS}

In this section, we prove Theorem 1.2 The (tame) blocks having Klein four defect groups are classified, for example, in [2, §6.6]. Throughout the remainder of this section, let $k$ have characteristic 2 , let $D$ be a Klein four group, and suppose $B$ is a block of $k G$ with defect group $D$. Then the number of simple $B$-modules, up to isomorphism, is either 1 or 3 . By $[2, \S 6.6], B$ has one of the following three forms:

(I) $B$ is isomorphic to $\operatorname{Mat}_{n}(k D)$ for some $n$, or

(II) $B$ is Morita equivalent to the group ring $k A_{4}$ of the alternating group of degree 4 , or

(III) $B$ is Morita equivalent to the principal block $B_{0}\left(k A_{5}\right)$ of the group ring $k A_{5}$ of the alternating group of degree 5 .

3.1. Case (I). We first look at $B=k D$. There is a unique simple $k D$-module, up to isomorphism, namely, the trivial simple $k D$-module, which we will denote by $S$.

Lemma 3.1. Let $V$ be an indecomposable $k D$-module with $\underline{\operatorname{End}}_{k D}(V)=k$. Then $V \cong \Omega^{i}(S)$ for some integer $i$ and $R(D, V)=W D$.

Proof. By Mazur [16] we have $R(G, S)=W D$. By Lemma 2.2 it follows that $R\left(D, \Omega^{i}(S)\right)=W D$ for all $i$. Since $k$ has characteristic 2 and $D$ is an abelian 2-group, it follows from [11, Thm. 10.1] and from [8] that the only indecomposable $k D$-modules with stable endomorphism ring equal to $k$ are of the form $\Omega^{i}(S)$ for some integer $i$.

We now suppose that $B$ is a block of $k G$ which is isomorphic to $\operatorname{Mat}_{n}(k D)$ for some $n$. Then there is a unique simple $B$-module, up to isomorphism, which we will denote by $T$.

Proposition 3.2. Let $V$ be an indecomposable B-module with $\underline{\operatorname{End}}_{k G}(V)=k$. Then $V \cong \Omega^{i}(T)$ for some integer $i$ and $R(G, V)=W D$.

Proof. Since $B$ and $k D$ are Morita equivalent, the $B$-modules of the form $\Omega^{i}(T)$ are the only $B$-modules that have stable endomorphism ring equal to $k$. Let now $B_{W}$ be the block of $W G$ corresponding to $B$. Then $B_{W}$ and $W D$ are Morita equivalent by [15, Cor 1.4]. Since $S$ and $T$ correspond to each other under this Morita equivalence, Proposition 3.2 follows from Lemma 3.1 and Proposition 2.5.

3.2. Case (II). We first look at $B=k A_{4}$. There are 3 non-isomorphic simple $k A_{4}$-modules, which we will denote by $S_{0}, S_{1}, S_{2}$, where $S_{0}$ is the trivial simple $k A_{4}$-module.

Lemma 3.3. Let $V$ be an indecomposable $k A_{4}$-module with $\underline{\operatorname{End}}_{k A_{4}}(V)=k$. Then either

(a) $V \cong \Omega^{i}\left(S_{j}\right), j=0,1,2$, for some integer $i$ and $R\left(A_{4}, V\right)=W$, or

(b) $V$ is uniserial of length 2 and $R\left(A_{4}, V\right)=k$.

Proof. The simple $k A_{4}$-modules $S_{0}, S_{1}, S_{2}$ are the inflations of the simple $k(\mathbb{Z} / 3 \mathbb{Z})$ modules to $A_{4}$. Hence they are 1-dimensional over $k$, and it follows by Mazur [16] that the universal deformation ring of $S_{j}, j=0,1,2$, is the group ring over $W$ of the maximal abelian 2-quotient of $A_{4}$. Thus $R\left(A_{4}, S_{j}\right)=W$ for $j=0,1,2$. By Lemma 2.2(ii), it follows that $R\left(A_{4}, \Omega^{i}\left(S_{j}\right)\right)=W$ for $j=0,1,2$ and for all $i$. 
We have 6 uniserial $k A_{4}$-modules of length 2 , namely, the $k A_{4}$-modules $U_{i j}$ that have descending composition series $\left(S_{i}, S_{j}\right)$, where $i \neq j$ is in $\{0,1,2\}$. It is obvious that the stable endomorphism ring of $U_{i j}$ is $k$. Since $\operatorname{Ext}_{k A_{4}}^{1}\left(U_{i j}, U_{i j}\right)=0$, it follows that $R\left(A_{4}, U_{i j}\right)$ is a quotient of $W$. We now show that $R\left(A_{4}, U_{i j}\right)=k$. The 6 uniserial modules $U_{i j}$ are in the $\Omega$-orbit of either $U_{12}$ or $U_{21}$. Hence it is enough to show that $R\left(A_{4}, U_{12}\right)=k=R\left(A_{4}, U_{21}\right)$. We use that $A_{4}$ is a subgroup of $A_{5}$ of index 5 . There are two simple 2-dimensional $k A_{5}$-modules $Z_{1}$ and $Z_{2}$ which define two isomorphisms

$$
\tau_{i}: A_{5} \rightarrow \mathrm{SL}_{2}\left(\mathbb{F}_{4}\right) \subset \mathrm{GL}_{2}\left(\mathbb{F}_{4}\right)
$$

for $i=1,2$. The Borel subgroup $S T$ of $\mathrm{SL}_{2}\left(\mathbb{F}_{4}\right)$ consisting of upper triangular matrices is isomorphic to $A_{4}$. When we consider $\tau_{i}, i=1,2$, restricted to the full pre-image of $S T$ under $\tau_{i}$, we obtain two isomorphisms

$$
\rho_{i}: A_{4} \rightarrow S T \subset \mathrm{GL}_{2}\left(\mathbb{F}_{4}\right)
$$

for $i=1,2$, which correspond to two uniserial $\mathbb{F}_{4} A_{4}$-modules of length 2 . Since $\mathbb{F}_{4}$ is a splitting field for $A_{4}$, it follows that $\rho_{1}, \rho_{2}$ can be identified with two uniserial $k A_{4}$-modules. Because the images of $\rho_{i}, i=1,2$, are in $\mathrm{SL}_{2}\left(\mathbb{F}_{4}\right)$, we conclude that these two uniserial $k A_{4}$-modules are $U_{12}$ and $U_{21}$. We now use the argumentation of [4, Section 6] to determine the universal deformation rings of these modules. Let $\tilde{A}_{5}$ be the double cover of $A_{5}$, i.e., $\tilde{A}_{5}=\mathrm{SL}_{2}\left(\mathbb{F}_{5}\right)$. Then $Z_{1}$ and $Z_{2}$ have lifts over $W$ when viewed as $k \tilde{A}_{5}$-modules. Let

$$
\lambda_{i}: \tilde{A}_{5} \rightarrow \mathrm{GL}_{2}(W / 4 W),
$$

$i=1,2$, be the corresponding lift of $Z_{i}$ over $W / 4 W$ and let $H_{i}$ be the image of $\lambda_{i}$. For $i=1,2$, we have a commutative diagram

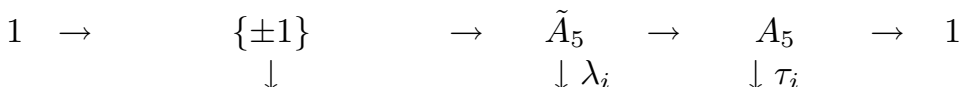

$$
\begin{aligned}
& 1 \rightarrow 1+2 \mathrm{Mat}_{2}(W / 4 W) \rightarrow H_{i} \rightarrow \mathrm{SL}_{2}\left(\mathbb{F}_{4}\right) \rightarrow 1 .
\end{aligned}
$$

In the bottom row of this diagram, one has an isomorphism

$$
\delta: 1+2 \operatorname{Mat}_{2}(W / 4 W) \rightarrow \operatorname{Mat}_{2}(k)^{+}
$$

sending $1+2 \alpha \bmod 4$ to $\alpha \bmod 2$, where $\operatorname{Mat}_{2}(k)^{+}$is the additive group of $\operatorname{Mat}_{2}(k)$. Let $\beta_{i} \in H^{2}\left(A_{5}, \mathrm{M}_{2}(k)^{+}\right)$be the extension class defined by the bottom row of (3.3) and the inverse of the map $\delta$ in (3.4). It follows from the proof of [4, Prop. 6.1] that the extension class $\beta_{i}$ is nontrivial for $i=1,2$. We define $\gamma_{i}$ to be the restriction $\operatorname{Res}_{A_{5}}^{A_{4}}\left(\beta_{i}\right), i=1,2$. Then $\gamma_{i} \in H^{2}\left(A_{4}, \operatorname{Mat}_{2}(k)^{+}\right)$is the extension class defined by the inverse of the map $\delta$ in (3.4), and by the bottom row of the diagram

$$
\begin{aligned}
& 1 \rightarrow 1+2 \operatorname{Mat}_{2}(W / 4 W) \rightarrow H_{i} \rightarrow \mathrm{SL}_{2}\left(\mathbb{F}_{4}\right) \rightarrow 1
\end{aligned}
$$

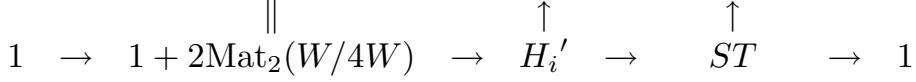

where the top row is the bottom row of Diagram (3.3) and $H_{i}{ }^{\prime}$ is the full preimage of $S T$ in $H_{i}$. The existence of a lift of $U_{12}$, respectively $U_{21}$, over $W / 4 W$ is equivalent to the splitting of the bottom row of (3.5), i.e., to the triviality of $\gamma_{i}$, for $i=1,2$. By [22, Prop. VIII.3.4], we have

$$
\operatorname{Cores}_{A_{4}}^{A_{5}}\left(\operatorname{Res}_{A_{5}}^{A_{4}}\left(\beta_{i}\right)\right)=\left[A_{5}: A_{4}\right]=5 .
$$


Hence, since 5 is relatively prime to $2=\operatorname{char}(k)$, we conclude that $\gamma_{i}=\operatorname{Res}_{A_{5}}^{A_{4}}\left(\beta_{i}\right)$ is nontrivial. This means that $R\left(A_{4}, U_{i j}\right)=k$ for $i \neq j$ in $\{0,1,2\}$.

It remains to show that there are no other indecomposable $k A_{4}$-modules $V$ that have stable endomorphism ring equal to $k$. We use that $k A_{4} \cong k \mathcal{Q} / I$ as $k$-algebras, where the quiver $\mathcal{Q}$ and the ideal $I$ of the path algebra $k \mathcal{Q}$ are given as (see [13. Cor. V.2.4.1])

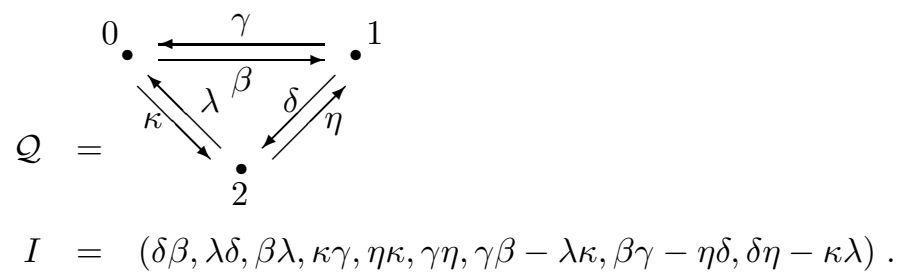

According to [13. Lemma II.7.4], the remaining indecomposable $k A_{4}$-modules lie either in a 3-tube, but not at the mouth of the 3-tube, or in a 1-tube of the stable Auslander-Reiten quiver of $k A_{4}$. We first look at the case of a module $X$ lying in a 3 -tube, but not at the mouth of it. The modules at the mouth are exactly the uniserial modules $U_{i j}$, where $i \neq j$ in $\{0,1,2\}$. There are $k$-algebra automorphisms of $k A_{4}$ that interchange the two 3-tubes, respectively permute the modules at the mouth of a given 3-tube. Thus it is enough to show that the stable endomorphism ring is not $k$ of a module $X$ of length $2 n \geq 4$ which has the following form (where we use the notation of [13 p. 62]):

$$
X=\begin{array}{lllllll}
S_{0} & S_{2} & S_{1} & S_{0} & \cdots & S_{i} \\
S_{1} & S_{0} & S_{2} & S_{1} & S_{j}
\end{array}
$$

where $\left(S_{i}, S_{j}\right)$ are any of $\left\{\left(S_{0}, S_{1}\right),\left(S_{2}, S_{0}\right),\left(S_{1}, S_{2}\right)\right\}$. Recall that $S_{0}, S_{1}$ and $S_{2}$ are 1-dimensional over $k$. Since $X$ has length at least 4 , it is enough to show that an endomorphism $\xi$ of $X$ that sends the first module $S_{0}$ in the top isomorphically to the second module $S_{0}$ in the socle does not factor through a projective module. By [14, the endomorphisms of $X$ that factor through a projective $k A_{4}$-module are linear combinations of three types of endomorphisms: (i) endomorphisms that send two consecutive modules in the top of $X$ simultaneously to two consecutive modules in the socle of $X$, (ii) endomorphisms that send a module $S_{1}$ in the top of $X$ to the first module $S_{1}$ in the socle of $X$, and (iii) endomorphisms that send the last module $S_{i}$ in the top of $X$ to a module $S_{i}$ in the socle of $X$. It follows from this description that the endomorphism $\xi$ of $X$ cannot factor through a projective module.

We now consider the indecomposable $k A_{4}$-modules that lie in 1-tubes. By [13. II.7.0], these modules are all band modules. Since the only band is $\beta \kappa^{-1} \delta \gamma^{-1} \lambda \eta^{-1}$, we call these band modules $M(\mu, n)$, where $n$ is a positive integer and $\mu \in k \backslash\{0\}$. Then $M(\mu, n)$ is $6 n$-dimensional over $k$, and we have the following actions:

$$
\beta \text { acts as }\left(\begin{array}{cccccc}
0 & J_{n}(\mu) & 0 & 0 & 0 & 0 \\
0 & 0 & 0 & 0 & 0 & 0 \\
0 & 0 & 0 & 0 & 0 & 0 \\
0 & 0 & 0 & 0 & 0 & 0 \\
0 & 0 & 0 & 0 & 0 & 0 \\
0 & 0 & 0 & 0 & 0 & 0
\end{array}\right), \kappa \text { acts as }\left(\begin{array}{cccccc}
0 & 0 & 0 & 0 & 0 & 0 \\
0 & 0 & 0 & 0 & 0 & 0 \\
0 & I_{n} & 0 & 0 & 0 & 0 \\
0 & 0 & 0 & 0 & 0 & 0 \\
0 & 0 & 0 & 0 & 0 & 0 \\
0 & 0 & 0 & 0 & 0 & 0
\end{array}\right) \text {, }
$$




$$
\begin{aligned}
& \delta \text { acts as }\left(\begin{array}{cccccc}
0 & 0 & 0 & 0 & 0 & 0 \\
0 & 0 & 0 & 0 & 0 & 0 \\
0 & 0 & 0 & I_{n} & 0 & 0 \\
0 & 0 & 0 & 0 & 0 & 0 \\
0 & 0 & 0 & 0 & 0 & 0 \\
0 & 0 & 0 & 0 & 0 & 0
\end{array}\right), \gamma \text { acts as }\left(\begin{array}{cccccc}
0 & 0 & 0 & 0 & 0 & 0 \\
0 & 0 & 0 & 0 & 0 & 0 \\
0 & 0 & 0 & 0 & 0 & 0 \\
0 & 0 & 0 & 0 & 0 & 0 \\
0 & 0 & 0 & I_{n} & 0 & 0 \\
0 & 0 & 0 & 0 & 0 & 0
\end{array}\right) \text {, } \\
& \lambda \text { acts as }\left(\begin{array}{cccccc}
0 & 0 & 0 & 0 & 0 & 0 \\
0 & 0 & 0 & 0 & 0 & 0 \\
0 & 0 & 0 & 0 & 0 & 0 \\
0 & 0 & 0 & 0 & 0 & 0 \\
0 & 0 & 0 & 0 & 0 & I_{n} \\
0 & 0 & 0 & 0 & 0 & 0
\end{array}\right), \eta \text { acts as }\left(\begin{array}{cccccc}
0 & 0 & 0 & 0 & 0 & I_{n} \\
0 & 0 & 0 & 0 & 0 & 0 \\
0 & 0 & 0 & 0 & 0 & 0 \\
0 & 0 & 0 & 0 & 0 & 0 \\
0 & 0 & 0 & 0 & 0 & 0 \\
0 & 0 & 0 & 0 & 0 & 0
\end{array}\right) \text {, }
\end{aligned}
$$

where $J_{n}(\mu)$ is the $n \times n$ Jordan block with eigenvalue $\mu$, and $I_{n}$ is the $n \times n$ identity matrix. According to [14, the endomorphisms of $M(\mu, n)$ are all $6 n \times 6 n$ matrices of the form

$$
\left(\begin{array}{cccccc}
A & 0 & 0 & E & 0 & 0 \\
0 & A & 0 & 0 & 0 & 0 \\
0 & 0 & A & 0 & 0 & D \\
0 & 0 & 0 & A & 0 & 0 \\
0 & C & 0 & 0 & A & 0 \\
0 & 0 & 0 & 0 & 0 & A
\end{array}\right)
$$

so that $C, D, E$ are arbitrary $n \times n$ matrices, and $A$ is an upper triangular $n \times n$ matrix with equal entries in each diagonal. Hence $\operatorname{dim}_{k} \operatorname{End}_{k A_{4}}(M(\mu, n))=3 n^{2}+n$. A matrix calculation shows that all endomorphisms $\Theta_{i}$ of $M(\mu, n)$ factoring through the projective indecomposable module $P\left(S_{i}\right), i=0,1,2$, have the form

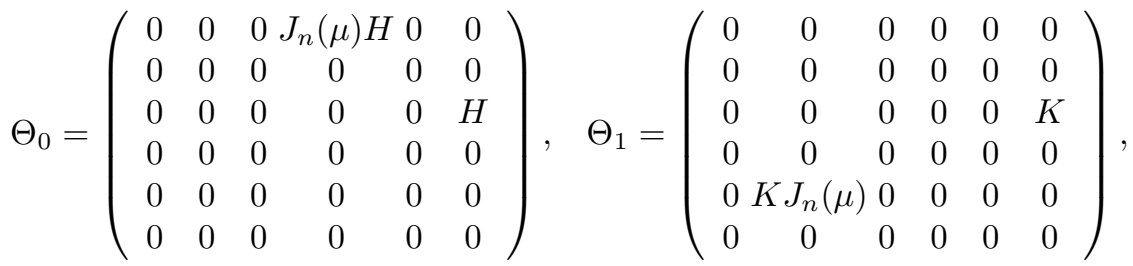

$$
\begin{aligned}
& \Theta_{2}=\left(\begin{array}{cccccc}
0 & 0 & 0 & L & 0 & 0 \\
0 & 0 & 0 & 0 & 0 & 0 \\
0 & 0 & 0 & 0 & 0 & 0 \\
0 & 0 & 0 & 0 & 0 & 0 \\
0 & L & 0 & 0 & 0 & 0 \\
0 & 0 & 0 & 0 & 0 & 0
\end{array}\right)
\end{aligned}
$$

where $H, K, L$ are $n \times n$ matrices. The matrices of type $\Theta_{2}$ which are sums of matrices of type $\Theta_{0}$ and $\Theta_{1}$ are exactly those for which $H=K$ and $J_{n}(\mu) H=$ $K J_{n}(\mu)$. This yields matrices of type $\Theta_{2}$ for which $L$ is an upper triangular matrix with equal entries in each diagonal. Thus $\operatorname{dim}_{k} \underline{\operatorname{End}}_{k A_{4}}(M(\mu, n))=\left(3 n^{2}+n\right)-$ $\left(3 n^{2}-n\right)=2 n$. This completes the proof of Lemma 3.3.

We now consider the case that $B$ is a block of $k G$ that is Morita equivalent to $k A_{4}$. Then there are 3 non-isomorphic simple $B$-modules, which we will denote by $T_{0}, T_{1}, T_{2}$ so that $S_{j}$ corresponds to $T_{j}$ under the Morita equivalence. 
Proposition 3.4. Let $V$ be an indecomposable B-module with $\underline{\operatorname{End}}_{k G}(V)=k$. Then either

(a) $V \cong \Omega^{i}\left(T_{j}\right), j=0,1,2$, for some integer $i$ and $R(G, V)=W$, or

(b) $V$ is uniserial of length 2 and $R(G, V)=k$.

Proof. Since $B$ and $k A_{4}$ are Morita equivalent, the only $B$-modules with stable endomorphism ring equal to $k$ are either of the form $\Omega^{i}\left(T_{j}\right), j=0,1,2$, or they are uniserial of length 2. Let now $B_{W}$ be the block of $W G$ corresponding to $B$. Then $B_{W}$ and $W A_{4}$ are Morita equivalent by [15, Cor. 1.4]. Since $S_{j}$ and $T_{j}$ (respectively uniserial modules of length 2) correspond to each other under this Morita equivalence, Proposition 3.4 follows from Lemma 3.3 and Proposition 2.5.

3.3. Case (III). We first assume that $B$ is the principal block $B_{0}\left(k A_{5}\right)$ of $k A_{5}$. There are 3 non-isomorphic simple $B_{0}\left(k A_{5}\right)$-modules, which we will denote by $S_{0}, S_{1}, S_{2}$, so that $S_{0}$ is the trivial simple $k A_{5}$-module. Further, $B_{0}\left(k A_{5}\right)$ has 4 non-isomorphic uniserial modules of length 2 , which we will denote by

$$
U_{01}=\begin{aligned}
& S_{0} \\
& S_{1}
\end{aligned}, U_{02}=\begin{aligned}
& S_{0} \\
& S_{2}
\end{aligned}, U_{10}=\begin{aligned}
& S_{1} \\
& S_{0}
\end{aligned}, U_{20}=\begin{gathered}
S_{2} \\
S_{0}
\end{gathered} .
$$

Lemma 3.5. Let $V$ be an indecomposable $B_{0}\left(k A_{5}\right)$-module with $\underline{\operatorname{End}}_{k A_{5}}(V)=k$. Then either

(a) $V \cong \Omega^{i}\left(S_{0}\right)$ for some integer $i$ and $R\left(A_{5}, V\right)=W$, or

(b) $V \cong \Omega^{i}\left(U_{j}\right), j=01,02,10,20$, for some integer $i$ and $R\left(A_{5}, V\right)=W$, or

(c) $V \cong \Omega^{i}\left(S_{j}\right), j=1,2$ and $i=0,2,4$, and $R\left(A_{5}, V\right)=k$.

Proof. It follows from Mazur 16 that the universal deformation ring of $S_{0}$ is $R\left(A_{5}, S_{0}\right)=W$. By [4, Prop. 6.1], the universal deformation ring of $S_{j}, j=1,2$, is $R\left(A_{5}, S_{j}\right)=k$. Note that $S_{j}, j=1,2$, lie at the mouths of 3 -tubes in the stable Auslander-Reiten quiver of $B_{0}\left(k A_{5}\right)$.

We now look at $U_{j}, j=01,02,10,20$. Since $\operatorname{Ext}_{k A_{5}}^{1}\left(U_{j}, U_{j}\right)=0$, the universal deformation ring $R\left(A_{5}, U_{j}\right)$ is a quotient of $W$. Because $\Omega^{-2}\left(U_{01}\right)=U_{20}$ and $\Omega^{-2}\left(U_{02}\right)=U_{10}$, we now concentrate on $j=01,02$. Let $F$ be the quotient field of $W$. The decomposition matrix corresponding to the principal block $B_{0}\left(k A_{5}\right)$ looks as follows (see [13, p. 295]):

\begin{tabular}{c|ccc} 
& $S_{0}$ & $S_{1}$ & $S_{2}$ \\
\hline$X_{0}$ & 1 & 0 & 0 \\
$X_{1}$ & 1 & 1 & 0 \\
$X_{2}$ & 1 & 0 & 1 \\
$X_{3}$ & 1 & 1 & 1
\end{tabular}

where $X_{0}, X_{1}, X_{2}, X_{3}$ are the irreducible $F A_{5}$-modules belonging to $B_{0}\left(k A_{5}\right)$. Since the exponent of $A_{5}$ is not a multiple of $4, F$ is a splitting field for $A_{5}$ and all its subgroups. Let $P_{0}$ be a lift over $W$ of the $k A_{5}$-projective cover of $S_{0}$. By [10, Thm. 18.26], the $F$-character of $P_{0}$ is given by $F \otimes_{W} P_{0}=X_{0} \oplus X_{1} \oplus X_{2} \oplus X_{3}$. Hence the $F$-character of $X_{1}$ lies inside the $F$-character of $P_{0}$. By [24, Thm. 1 and Cor.], there exists a pure submodule $Q_{1}$ of $P_{0}$ so that $P_{0} / Q_{1}$ affords the $F$-character of $X_{1}$, and $\left(P_{0} / Q_{1}\right) / 2\left(P_{0} / Q_{1}\right)$ is indecomposable. Since the top of $P_{0}$ is $S_{0}$, it follows that $\left(P_{0} / Q_{1}\right) / 2\left(P_{0} / Q_{1}\right) \cong U_{01}$. Thus $P_{0} / Q_{1}$ is a lift of $U_{01}$ over $W$. Analogously, we obtain a lift $P_{0} / Q_{2}$ of $U_{02}$ over $W$. This means that $R\left(A_{5}, U_{j}\right)=W$. 
Restriction from $A_{5}=\mathrm{SL}_{2}\left(\mathbb{F}_{4}\right)$ to its Borel subgroup $S T \cong A_{4}$ of upper triangular matrices induces a stable equivalence between $B_{0}\left(k A_{5}\right)$ and $k A_{4}$ (see, for example, [20, Example 1]). Note that $\operatorname{Res}_{A_{5}}^{A_{4}}\left(S_{0}\right)$ is the trivial simple $k A_{4}$-module, and $\operatorname{Res}_{A_{5}}^{A_{4}}\left(S_{i}\right), i=1,2$, is a uniserial $k A_{4}$-module of length 2. More precisely, there exists an ordering of the two nontrivial simple $k A_{4}$-modules $Y_{1}$ and $Y_{2}$ so that $\operatorname{Res}_{A_{5}}^{A_{4}}\left(S_{1}\right)=\begin{aligned} & Y_{2} \\ & Y_{1}\end{aligned}$ and $\operatorname{Res}_{A_{5}}^{A_{4}}\left(S_{2}\right)=\begin{aligned} & Y_{1} \\ & Y_{2}\end{aligned}$. It follows that $\operatorname{Res}_{A_{5}}^{A_{4}}\left(U_{01}\right)=\Omega\left(Y_{1}\right)$, $\operatorname{Res}_{A_{5}}^{A_{4}}\left(U_{02}\right)=\Omega\left(Y_{2}\right), \operatorname{Res}_{A_{5}}^{A_{4}}\left(U_{10}\right)=\Omega^{-1}\left(Y_{2}\right)$, and $\operatorname{Res}_{A_{5}}^{A_{4}}\left(U_{20}\right)=\Omega^{-1}\left(Y_{1}\right)$. Hence it follows from Lemma 3.3 that the modules in parts (a), (b) and (c) of the statement of Lemma 3.5 are the only indecomposable $B_{0}\left(k A_{5}\right)$-modules that have stable endomorphism ring equal to $k$. This completes the proof of Lemma 3.5

We now consider the case that $B$ is a block of $k G$ that is Morita equivalent to the principal block $B_{0}\left(k A_{5}\right)$. Then there are 3 non-isomorphic simple $B$-modules, which we will denote by $T_{0}, T_{1}, T_{2}$ so that $S_{j}$ corresponds to $T_{j}$ under the Morita equivalence. Further, there are 4 non-isomorphic uniserial $B$-modules of length 2, which we will denote by

$$
U_{01}{ }^{\prime}=\begin{aligned}
& T_{0} \\
& T_{1}
\end{aligned}, U_{02}{ }^{\prime}=\begin{aligned}
& T_{0} \\
& T_{2}
\end{aligned}, U_{10}{ }^{\prime}=\begin{aligned}
& T_{1} \\
& T_{0}
\end{aligned}, U_{20}{ }^{\prime}=\begin{aligned}
& T_{2} \\
& T_{0}
\end{aligned} .
$$

Proposition 3.6. Let $V$ be an indecomposable B-module with $\underline{\operatorname{End}}_{k G}(V)=k$. Then either

(a) $V \cong \Omega^{i}\left(T_{0}\right)$ for some integer $i$ and $R(G, V)=W$, or

(b) $V \cong \Omega^{i}\left(U_{j}{ }^{\prime}\right), j=01,02,10,20$, for some integer $i$ and $R(G, V)=W$, or

(c) $V \cong \Omega^{i}\left(T_{j}\right), j=1,2$ and $i=0,2,4$, and $R(G, V)=k$.

Proof. Since $B$ and $B_{0}\left(k A_{5}\right)$ are Morita equivalent, the only $B$-modules with stable endomorphism ring equal to $k$ are the modules occurring in parts (a), (b) and (c) of the statement of Proposition 3.6. Let now $B_{W}$ be the block of $W G$ corresponding to $B$. Then $B_{W}$ and $B_{0}\left(W A_{5}\right)$ are Morita equivalent by [15. Cor. 1.4]. Since $S_{j}$ and $T_{j}$ (respectively $U_{j}$ and $U_{j}{ }^{\prime}$ ) correspond to each other under this Morita equivalence, Proposition 3.6 follows from Lemma 3.5 and Proposition 2.5.

Theorem 1.2 follows from Lemma 2.3 and Propositions 3.2 , 3.4 and 3.6

Remark 3.7. It was shown in 18 that $W A_{4}$ and $B_{0}\left(W A_{5}\right)$ are splendidly derived equivalent. This means that the derived equivalence is given by a split endomorphism two-sided tilting complex of summands of permutation $\left(A_{4} \times A_{5}\right)$-modules induced from diagonal subgroups of $P \times P$, where $P$ is a common Sylow 2-subgroup of $A_{4}$ and $A_{5}$.

Note that the same universal deformation rings occur for modules $V$ of these blocks over $k$ having stable endomorphisms equal to $k$. Moreover, the stable Auslander-Reiten quivers of $k A_{4}$ and of $B_{0}\left(k A_{5}\right)$ are isomorphic as graphs. The modules which lie at the same position in the respective stable Auslander-Reiten quivers have isomorphic universal deformation rings.

\section{The symmetric group $S_{4}$ OF Degree 4}

Let $k$ have characteristic 2 . We want to consider the irreducible $k S_{4}$-modules and determine their universal deformation rings. The principal block of $k S_{4}$ is $k S_{4}$ itself, which has as defect groups dihedral groups of order 8. In particular, the defect groups are not abelian. We need the following result. 
Lemma 4.1. Let $R$ be a $W$-algebra that surjects onto $W$ so that $R / 2 R \cong k[t] /\left(t^{2}\right)$. Then either $R$ is isomorphic to a $W$-subalgebra of $W(\mathbb{Z} / 2 \mathbb{Z})$, or $R$ is isomorphic to $W[t] /\left(t^{2}\right)$.

Proof. It follows from the assumptions that $R$ is a quotient ring of a power series ring $W[[s]]$ in one variable. Hence we obtain a short exact sequence

$$
0 \rightarrow J \rightarrow W[[s]] \stackrel{\tau}{\longrightarrow} R \rightarrow 0 .
$$

We first show that we can choose $s$ so that $J \subseteq(s)$. Since $R$ surjects onto $W$ and $\tau: W[[s]] \rightarrow R$ is surjective, we obtain a short exact sequence

$$
0 \rightarrow K \rightarrow W[[s]] \stackrel{\psi}{\longrightarrow} W \rightarrow 0
$$

with $J \subseteq K$. On the other hand, $K=\operatorname{Ker}(\psi)$ is generated by a linear polynomial of the form $a s+b$ where $a$ is a unit in $W$. So $K=(s+c)$ with $c=a^{-1} b$. If we replace $s$ by $s+c$, then we can assume that $K=(s)$, and thus $J \subseteq(s)$. Next we show that $J+2 W[[s]]=\left(s^{2}, 2\right)$ as ideals. It follows from the assumptions that $J+2 W[[s]] \supseteq\left(s^{2}, 2\right)$. Now let $x \in J$; so $x=a_{1} s+s^{2} f(s)$ where $f$ is some element of $W[[s]]$. We have to show that $a_{1}$ is a multiple of 2 . If this were not the case, $a_{1}$ would be a unit in $W$, and thus $u=a_{1}+s f(s)$ would be a unit in $W[[s]]$. Hence $x=s \cdot u$, which is impossible since $R / 2 R \cong k[t] /\left(t^{2}\right)$.

Using the assumptions on $R=W[[s]] / J$ and $J \subset(s)$, it follows now that $J$ is generated by a square polynomial of the form $s^{2}+r s$ where $r \in W$ is a multiple of 2. If $r=0$, this implies that $R=W[[s]] /\left(s^{2}\right) \cong W[t] /\left(t^{2}\right)$. Now suppose $r \neq 0$. Then we define a $W$-algebra homomorphism $\rho$ by

$$
\begin{aligned}
\rho: R=W[[s]] /(s(s+r)) & \longrightarrow W \times W \\
\overline{1} & \mapsto(1,1) \\
\bar{s} & \mapsto(-r, 0)
\end{aligned}
$$

which is injective. Let $(x, y)$ be in the image of $\rho$, i.e.,

$$
(x, y)=\rho\left(b_{0} \bar{s}+b_{1}\right)=\left(b_{1}-b_{0} r, b_{1}\right) .
$$

Then $y-x=b_{0} r$ is a multiple of 2 . Hence the image of $\rho$ is a $W$-subalgebra of

$$
\{(x, y) \in W \times W \mid x \equiv y \bmod 2 W\} \cong W(\mathbb{Z} / 2 \mathbb{Z}) .
$$

There are two non-isomorphic simple $k S_{4}$-modules, given by the trivial $k S_{4^{-}}$ module $E_{0}$ and by a module $E_{1}$ of $k$-dimension 2 . Both $E_{0}$ and $E_{1}$ are inflated from irreducible $k S_{3} \cong k\left(S_{4} / K\right)$-modules, where $K$ denotes the normal subgroup of $S_{4}$ isomorphic to a Klein four group. In fact, $E_{1}$ is irreducible and projective as a $k S_{3}$-module.

Proposition 4.2. The universal deformation rings of $E_{0}$ and $E_{1}$ as $k S_{4}$-modules are

$$
R\left(S_{4}, E_{0}\right)=W(\mathbb{Z} / 2 \mathbb{Z}) \quad \text { and } \quad R\left(S_{4}, E_{1}\right)=W[t] /\left(t^{2}\right) .
$$

Proof. It follows from [16] that $R\left(S_{4}, E_{1}\right)=W S_{4}^{a b, 2}=W(\mathbb{Z} / 2 \mathbb{Z})$. Viewing $E_{1}$ as a $k S_{3}$-module leads to $R\left(S_{3}, E_{1}\right)=W$ by Lemma 2.2(iii). Hence, since $S_{3}$ is a quotient group of $S_{4}, R\left(S_{4}, E_{1}\right)$ surjects onto $R\left(S_{3}, E_{1}\right)=W$. 
Denote $R\left(S_{4}, E_{1}\right) / 2 R\left(S_{4}, E_{1}\right)$ by $R^{\prime}$. We now show that $R^{\prime} \cong k[t] /\left(t^{2}\right)$. By using, for example, the $k$-algebra in [13] p. 63], which is Morita equivalent to $k S_{4}$ by [13, Cor. V.2.5.1], we get

$$
\operatorname{Ext}_{k S_{4}}^{1}\left(E_{1}, E_{1}\right)=k
$$

which implies $R^{\prime}=k[t] /\left(t^{r}\right)$ for some $r$. There is a uniserial $k S_{4}$-module $M$ that is given as $M=\begin{gathered}E_{1} \\ E_{1}\end{gathered}$. If we let $t$ act as the shift down by one, it follows that $M$ is a free $k[t] /\left(t^{2}\right)$-module of rank 2 which is a lift of $E_{1}$ over $k[t] /\left(t^{2}\right)$. Hence there exists a $k$-algebra homomorphism

$$
\phi: R^{\prime} \rightarrow k[t] /\left(t^{2}\right)
$$

corresponding to $M$. Since $M$ is indecomposable as a $k S_{4}$-module, it follows that $\phi$ is surjective. Suppose $\phi$ is not a $k$-algebra isomorphism. Then there exists a surjective $k$-algebra homomorphism $\phi_{1}: R^{\prime} \rightarrow k[t] /\left(t^{3}\right)$ so that $\pi \phi_{1}=\phi$ where $\pi: k[t] /\left(t^{3}\right) \rightarrow k[t] /\left(t^{2}\right)$ is the natural projection. Let $M_{1}$ be a lift of $E_{1}$ over $k[t] /\left(t^{3}\right)$. Then $M_{1}$ is a lift of $M$ over $k[t] /\left(t^{3}\right)$ and $t^{2} M_{1} \cong E_{1}$. Hence we have a short exact sequence of $k[t] /\left(t^{3}\right) S_{4}$-modules

$$
0 \rightarrow t^{2} M_{1} \rightarrow M_{1} \rightarrow M \rightarrow 0 .
$$

Suppose this sequence splits as a sequence of $k S_{4}$-modules. Then $M \cong E_{1} \oplus M$ as $k S_{4}$-modules, and $t$ acts on $\left(\begin{array}{l}\lambda \\ v\end{array}\right) \in E_{1} \oplus M \cong M_{1}$ as multiplication by

$$
U_{t}=\left(\begin{array}{cc}
0 & \alpha \\
0 & \mu_{t}
\end{array}\right)
$$

where $\alpha: M \rightarrow E_{1}$ is a surjective $k S_{4}$-module homomorphism, and $\mu_{t}$ is the multiplication by $t$ on $M$. Since $t^{2} M_{1} \cong E_{1}$, there exists a nonzero $\left(\begin{array}{l}\lambda \\ v\end{array}\right) \in$ $E_{1} \oplus M \cong M_{1}$ so that $U_{t}^{2}\left(\begin{array}{l}\lambda \\ v\end{array}\right)$ is not zero. But

$$
U_{t}^{2}\left(\begin{array}{l}
\lambda \\
v
\end{array}\right)=\left(\begin{array}{c}
\alpha\left(\mu_{t}(v)\right) \\
\mu_{t}^{2}(v)
\end{array}\right)=\left(\begin{array}{l}
0 \\
0
\end{array}\right)
$$

which gives a contradiction. Hence the short exact sequence (4.6) does not split as a sequence of $k S_{4}$-modules. Therefore, $M_{1}$ is an indecomposable $k S_{4}$-module. The description of the indecomposable $k S_{4}$-modules as string and band modules (see, for example, [13, §II.3] using [13, Cor. V.2.5.1]), shows that an indecomposable $k S_{4}$-module of the form $M_{1}$ does not exist. Hence $\phi: R^{\prime} \rightarrow k[t] /\left(t^{2}\right)$ must be a $k$-algebra isomorphism.

By Lemma 4.1. it follows that either $R\left(S_{4}, E_{1}\right) \cong W[t] /\left(t^{2}\right)$, or $R\left(S_{4}, E_{1}\right)$ is isomorphic to a subquotient ring of $W(\mathbb{Z} / 2 \mathbb{Z})$. If the latter is the case, the proof of Lemma 4.1 shows that

$$
R\left(S_{4}, E_{1}\right) \cong W[[s]] /\left(s\left(s+2 r_{1}\right)\right)=W[[s]] /\left(\left(s+r_{1}\right)^{2}-r_{1}^{2}\right) \cong W[[t]] /\left(t^{2}-r_{1}^{2}\right)
$$

for some nonzero $r_{1} \in W$. Because there are two different surjective $W$-algebra homomorphisms $R\left(S_{4}, E_{1}\right) \rightarrow W$, there are two non-isomorphic lifts of $E_{1}$ as a 
$k S_{4}$-module over $W$. We show now that this is not possible. Let $F$ be the quotient field of $W$. Then

$$
\begin{aligned}
F S_{4} & \cong F \times F \times \operatorname{Mat}_{2}(F) \times \operatorname{Mat}_{3}(F) \times \operatorname{Mat}_{3}(F), \\
F\left(S_{4} / K\right) \cong F S_{3} & \cong F \times F \times \operatorname{Mat}_{2}(F) .
\end{aligned}
$$

Since $E_{1}$ is irreducible over $k S_{4}$, it follows for lifts $X$ of $E_{1}$ over $W$ that $F \otimes_{W} X$ is an irreducible $F S_{4}$-module of rank 2 . Since there is only one matrix ring of rank 2 in the product giving $F S_{4}$ which corresponds to the matrix ring of rank 2 in the product of $F\left(S_{4} / K\right) \cong F S_{3}$, it follows that $X$ is actually a $W S_{3}$-module, i.e., it has trivial action by $K$. Hence all lifts of $E_{1}$ as a $k S_{4}$-module over $W$ are actually lifts of $E_{1}$ as a $k S_{3}$-module over $W$. Since $R\left(S_{3}, E_{1}\right)=W$, this implies that all lifts of $E_{1}$ as a $k S_{4}$-module over $W$ are isomorphic. Therefore, there is only one surjective $W$-algebra homomorphism $R\left(S_{4}, E_{1}\right) \rightarrow W$, and $R\left(S_{4}, E_{1}\right)=W[t] /\left(t^{2}\right)$.

Corollary 4.3. Question 1.1 has a positive answer in case $V$ is a simple $k S_{4}$ module.

Proof. We have to show that $W[t] /\left(t^{2}\right)$ is isomorphic to a subquotient ring of $W D_{8}$, where $D_{8}$ denotes a dihedral group of order 8 . Let $F$ be the quotient field of $W$. Then

$$
F D_{8} \cong F \times F \times F \times F \times \operatorname{Mat}_{2}(F) .
$$

Let $a \in \operatorname{Mat}_{2}(F)$ with $a^{2}=0$, and let $x \in F D_{8}$ be such that $x$ corresponds to $(0,0,0,0, a)$ in the above product. Then we can multiply $x$ with a large enough power of 2 to obtain an element $y \in W D_{8}$ with $y^{2}=0$. Hence $W[t] /\left(t^{2}\right)$ is isomorphic to a subquotient ring of $W D_{8}$.

Remark 4.4. Proposition 4.2 shows that Question 1.1 cannot be refined for nonabelian defect groups $D$ by replacing $D$ by its maximal abelian quotient.

\section{REFERENCES}

[1] J. L. Alperin, Local Representation Theory, Cambridge studies in advanced mathematics 11, Cambridge University Press, Cambridge, 1986. MR 87i:20002

[2] D. J. Benson, Representations and Cohomology I, Cambridge studies in advanced mathematics 30, Cambridge University Press, Cambridge, 1991. MR 92m:20005

[3] F. M. Bleher and T. Chinburg, Universal deformation rings and cyclic blocks, Math. Ann. 318 (2000), 805-836. MR 2001m:20013

[4] F. M. Bleher and T. Chinburg, Applications of versal deformations to Galois theory, accepted by Comment. Math. Helv., 2002.

[5] F. M. Bleher and T. Chinburg, Deformations and derived categories, C. R. Acad. Sci. Paris Ser. I Math. 334 (2002), no. 2, 97-100. CMP2002:08

[6] C. Breuil, B. Conrad, F. Diamond and R. Taylor, On the modularity of elliptic curves over $\mathbb{Q}$ : Wild 3-adic exercises, J. Amer. Math. Soc. 14 (2001), 843-939. MR 2002d:11058

[7] M. Broué, Isométries parfaites, types de blocs, catégories dérivées, Astérisque No. 181-182 (1990), 61-92. MR 91i:20006

[8] J. F. Carlson, A characterization of endotrivial modules over p-groups, Manuscripta Math. 97 (1998), no. 3, 303-307. MR 99h:20006

[9] G. Cornell, J. H. Silverman and G. Stevens (eds.), Modular Forms and Fermat's Last Theorem, Papers from the Instructional Conference on Number Theory and Arithmetic Geometry held at Boston University, Boston, MA, August 9-18, 1995, Springer-Verlag, New York-Berlin, 1997. MR 99k:11004

[10] C. Curtis and I. Reiner, Methods of Representation Theory, Vols. I and II, John Wiley and Sons, New York, 1981, 1987. MR 82i:20001; MR 88f:20002 
[11] E. Dade, Endo-permutation modules II, Ann. of Math. 108 (1978), no. 2, 317-345. MR 80a:13008b

[12] B. de Smit and H. W. Lenstra, Explicit construction of universal deformation rings, Modular Forms and Fermat's Last Theorem (Boston, MA, 1995), Springer-Verlag, New York, 1997, pp. 313-326. CMP 98:16

[13] K. Erdmann, Blocks of Tame Representation Type and Related Algebras, Lecture Notes in Mathematics, Vol. 1428, Springer-Verlag, Berlin-Heidelberg-New York, 1990. MR 91c:20016

[14] H. Krause, Maps between tree and band modules, J. Algebra 137 (1991), 186-194. MR 92j:16010

[15] M. Linckelmann, The source algebras of blocks with a Klein four defect group, J. Algebra 167 (1994), 821-854. MR 95h:20014

[16] B. Mazur, Deforming Galois representations, Galois groups over $\mathbb{Q}$ (Berkeley, CA, 1987), Springer-Verlag, New York, 1989, pp. 385-437. MR 90k:11057

[17] B. Mazur, Deformation theory of Galois representations, Modular Forms and Fermat's Last Theorem (Boston, MA, 1995), Springer-Verlag, New York, 1997, pp. 243-311.

[18] J. Rickard, Splendid equivalences: derived categories and permutation modules, Proc. London Math. Soc. (3) 72 (1996), 331-358. MR 97b:20011

[19] J. Rickard, The abelian defect group conjecture, Proceedings of the International Congress of Mathematicians, Vol. II (Berlin, 1998), Doc. Math., 1998, Extra Vol. II, pp. 121-128. MR 99f:20014

[20] R. Rouquier, From stable equivalences to Rickard equivalences for blocks with cyclic defect, Groups '93 Galway/St. Andrews, Vol. 2, London Math. Soc. Lecture Note Ser., 212, Cambridge Univ. Press, Cambridge, 1995, pp. 512-523. MR 96h:20021

[21] M. Schlessinger, Functors of Artin Rings, Trans. Amer. Math. Soc. 130 (1968), 208-222. MR 36:184

[22] J.-P. Serre, Corps locaux, Hermann, Paris, 1968. MR 50:7096

[23] R. L. Taylor and A. Wiles, Ring-theoretic properties of certain Hecke algebras, Ann. of Math. (2) 141 (1995), no. 3, 553-572. MR 96d:11072

[24] J. G. Thompson, Vertices and sources, J. Algebra 6 (1967), 1-6. MR 34:7677

[25] A. Wiles, Modular elliptic curves and Fermat's last theorem, Ann. of Math. (2) 141 (1995), no. 3, 443-551. MR 96d:11071

Department of Mathematics, University of Iowa, Iowa City, Iowa 52242-1419

E-mail address: fbleher@math.uiowa.edu 